\title{
Retrograde Intrarenal Surgery for the Management of Kidney Stones Smaller than 20 mm: A Single-Center Experience of 452 Cases
}

\author{
Ramazan Topaktaş, AC Ahmet Ürkmez
}

Department of Urology, University of Health Sciences, Hamidiye Faculty of Medicine, Haydarpasa Numune Health Application and Research Center, Istanbul, Turkey

\begin{abstract}
Introduction: This study presents our outcomes of 452 patients who underwent retrograde intrarenal surgery (RIRS) for the treatment of renal stones smaller than $20 \mathrm{~mm}$ in the last five years

Methods: We retrospectively reviewed the file of 452 patients who were performed RIRS for renal stones in our clinic between January 2013 and February 2018. Fluoroscopy was always used in all cases. Patients were investigated regarding demographic and operation data, stone location and size and stone-free rates. Patients were controlled by direct urinary system graphy on the postoperative first day and by non-contrasted computed tomography (CT) in a month after the intervention. Stone-free status was documented on CT if there were no residual stones or presence of residual fragments smaller than $3 \mathrm{~mm}$ after a month.

Results: There were 285 (63\%) male and 167 (36.9\%) female patients with a mean age of 47.9 (range 12-85) years. Mean stone size was $13.5 \mathrm{~mm}$ (range 7-20 mm). Ureteral access sheath was used in 388 (85.8\%) of the patients. Mean operative and fluoroscopy screening times were 70.6 (range 45-150) minutes and 17.6 (range 3-55) second, respectively. The average hospital stay was 1.3 (range 1-5) days. Twenty (4.4\%) patients had minor complications, including renal colic, hematuria, infection and/or fever. No major complications and blood transfusions were noted. Stone-free status was achieved in 403 (89.1\%) of the patients in the first month postoperatively

Discussion and Conclusion: We believe that RIRS is an effective, reliable and safe treatment modality for kidney stones smaller than $20 \mathrm{~mm}$. The procedure has low morbidity and high success rate

Keywords: Flexible ureterorenoscopy; renal stone; retrograde intrarenal surgery.
\end{abstract}

$\mathrm{K}$ idney stones are one of the most common diseases that cause patient suffering, loss of work, and morbidity with socioeconomic consequences ${ }^{[1]}$. The prevalence of urolithiasis has been noticed as $2.8 \%$ in the USA, $1.5 \%$ in Europe, and $14.8 \%$ in Turkey ${ }^{[2,3]}$. In addition, urinary system stone disease has a tendency for recurrence with a rate of $50 \%$ over 10 years.
The treatment of renal calculi has evolved considerably during the last four decades. In parallel with the progress of technology in the management of renal stones, lower morbidity and higher stone-free rates have been obtained. Previously, renal stones have been managed with open surgery, while, currently, many minimally invasive treatment techniques as electroshock wave lithotripsy (ESWL),

Correspondence (Illetişim): Ramazan Topaktaş, M.D. Saglik Bilimleri Universitesi Hamidiye Tip Fakultesi, Haydarpasa Numune Saglik Uygulama ve Arastirma Merkezi, Uroloji Anabilim Dali, Istanbul, Turkey

Phone (Telefon): +90 5059139561 E-mail (E-posta): ramazantopaktas@yahoo.com

Submitted Date (Başvuru Tarihi): 14.12.2018 Accepted Date (Kabul Tarihi): 22.12.2018

Copyright 2020 Haydarpaşa Numune Medical Journal

OPEN ACCESS This is an open access article under the CC BY-NC license (http://creativecommons.org/licenses/by-nc/4.0/) 
percutaneous nephrolithotomy (PCNL), retrograde intrarenal surgery (RIRS), and laparoscopic surgery can be applied in place of open surgery. The ideal treatment would be complete stone clearance in a single session with minimum trauma to the patients and prevention of new stone formation. The preference among the treatments depends on size, hardness and position of stone and experience of the surgeon ${ }^{[4,5]}$.

With advanced and highly qualified imaging modalities in technology, minimally invasive methods are all used more effectively for the management of renal stones. Technical improvements, including endoscope miniaturization, improved deflection mechanism, enhanced optical quality and tools, and introduction of disposables, have led to increased use of RIRS for renal stones. The purpose of all these technological developments is to provide the least morbidity and achieve no stone state. According to the European Association of Urology guidelines, the use of flexible ureterorenoscopy ( $f$-URS) as reliable and efficient alternative usually for the patients with obesity, complex anatomical kidney, musculoskeletal deformities, bleeding diathesis, unsuccessful ESWL procedure, and stones with size less than $2 \mathrm{~cm}{ }^{[6]}$. Although PCNL with enhanced morbidity for compact and rigid stones larger than $2 \mathrm{~cm}$ in diameter has been suggested as a first-line treatment, $\mathrm{f}$-URS can be performed as an alternative surgical treatment for this type of stone ${ }^{[6,7]}$.

In the present study, we have retrospectively analyzed and presented outcomes of 452 patients who underwent RIRS due to different size kidney stones in the last five years.

\section{Materials and Methods}

In this study, we retrospectively reviewed medical records of 452 patients who underwent RIRS for renal stones at Istanbul Haydarpasa Numune Training and Research Hospital from January 2013 to February 2018. This study was conducted in compliance with ethical principles defined in the Helsinki Declaration. Approval was obtained from the scientific committee of our hospital, and written informed consent was obtained from all participants and/or relatives before the operations. This study included patients with kidney stones with smaller than $20 \mathrm{~mm}$, ESWL-refractory, or previously failed ESWL, patients associated with musculoskeletal deformities and bleeding disorders. Demographic data of patients, size and site of stones, duration of operation, stone-free rates, and duration of hospital stay were investigated. All the patients were preoperatively evaluated using a physical examination, routine blood tests, coagulation tests, urinalysis, urine culture, direct urinary system graphy (KUB), urinary system ultrasound (US), non-contrasted computed tomography (CT) and/or intravenous pyelography (IVP). Patients with positive urine cultures were adequately treated with appropriate antibiotics, and all the patients had a negative urine culture before surgery. Stone size was determined by measuring the longest axis on the preoperative radiologic investigation. In patients with multiple renal stones, the stone size was calculated as the sum of the greatest dimensions of each stone.

\section{Surgical Technique}

Before the procedure, intravenous antibiotics (first-generation intravenous cephalosporin) were administrated. Under general anesthesia, firstly, patients were placed in the lithotomy position on an endoscopy table with fluoroscopic imaging capability. Then, under scopy, a hydraulic 0.035-inch safety guidewire was advanced upward inside the ureter. Semi-rigid ureterorenoscopy (9.5 Fr Karl Storz Ureterorensocope, Germany) was performed to exclude the presence of any ureteral surprise pathology, such as transitional cell carcinoma in the ureter and dilate the ureter. After two guidewires were advanced to the renal pelvis, a 9.5/11.5 F ureteral access sheath (UAS) was placed over the guidewire to sustain low intrarenal pressure and to simplify the extraction of stone fragments. Then, a 7.5 to $8.5 \mathrm{Fr}$ flexible ureterorenoscopy (FLEX-X2Karl Storz Endoscopy, Germany) was passed through UAS. F-URS was passed into the renal pelvis over the guidewire in the case of unsuccessful placement of UAS. When access could not be provided due to obstruction in ureteral orifice or other areas of the ureter, a Double-J stent was inserted, and operation was carried out after 2-4 weeks of the passive dilatation. Stones were fragmented with a $272 \mu \mathrm{m}$ holmium laser probe at an energy level of 0.6-1.0 J and frequency of $10-20 \mathrm{~Hz}$ until they were deemed small enough to pass spontaneously. Irrigation was delivered using a pressure pump with a regulated rate and pressure. At the end of the procedure, a fluoroscope was used to check for large residual fragments. Stone fragments larger than $3 \mathrm{~mm}$ were taken out using a basket catheter. A Double-J (4.8F) stent and $16 \mathrm{~F}$ Foley urethral catheters were inserted in all cases at the end of the procedure.

Patients were controlled on the first postoperative day by KUB after the removal of the urethral catheters. If necessary, patients treated with appropriate medical therapy and dietary suggestions were made postoperatively in the outpatient setting. Double-J stents were removed under 
local anesthesia 2-4 weeks after the operation. If available, stone analyses were performed. Stone-free status was determined by a low dose protocol spiral CT scan first month after the operation and success were defined as stonefree status or presence of insignificant residual fragments smaller than $3 \mathrm{~mm}$. In patients with residual calculi, the second stage RIRS or PCNL was performed. After the first month follow-up, patients were seen every six months during the first year and yearly thereafter.

The data were entered into the statistics program. All statistical analysis tests were performed using Statistical Package for the Social (SPSS Version 17.0 for Windows, SPSS Inc., Chicago, IL, USA). As descriptive statistics, frequencies, percentages, and mean values were calculated. Data were presented as mean (min-max) or number and percentage of the patients.

\section{Results}

A total of 452 patients (male, $n=285$; female, $n=167$ ) underwent RIRS operation. Mean age of female and male patients was 46.3 years (12-83) and 48.9 years (13-85), respectively. Patients' demographic data are summarized in Table 1.The mean stone size was $13.5 \mathrm{~mm}$ (range $7-20 \mathrm{~mm}$ ). The mean stone sizes were 13.6 (9-20) $\mathrm{mm}$ in lower, 15.8 (9-20) $\mathrm{mm}$ in middle, 14.8 (7-20) $\mathrm{mm}$ in upper poles, 17.4 (10-20) $\mathrm{mm}$ in renal pelvis, and 12.9 (8-20) $\mathrm{mm}$ in multiple calyceal stones. Stones located in lower $(n=116 ; 25.6 \%)$, middle $(n=49$; $10.8 \%)$, and upper $(n=46 ; 10.1 \%)$ poles, renal pelvis $(n=153$;

Table 1. Demographic datas of the patients

\begin{tabular}{lc}
\hline Parameters & Values $\mathbf{n}(\%)$ \\
\hline $\begin{array}{l}\text { Gender } \\
\text { Male }\end{array}$ & $285(63)$ \\
$\quad$ Female & $167(36.9)$ \\
Age, years & \\
$\quad<18$ & $10(2.2)$ \\
$\quad 18-65$ & $390(86.2)$ \\
$>65$ & $52(11.5)$ \\
Antithrombotic and/or antiaggregant use & \\
$\quad$ Yes & $25(5.5)$ \\
$\quad$ No & $427(94.4)$ \\
History of ESWL & $34(7.5)$ \\
Prior history of open surgery & $13(2.8)$ \\
$\quad$ Yes & $439(97.1)$ \\
$\quad$ No & $42(9.2)$ \\
Preoperative Double-J stent & \\
Degree of hydronephrosis before operation & $386(85.3)$ \\
$\quad$ None or mild & $66(14.6)$ \\
$\quad$ Moderate or severe &
\end{tabular}

$33.8 \%)$, and multiple calyces ( $n=88 ; 19.4 \%)$. Patients' stone characteristics are summarized in Table 2.

Bilateral RIRS was applied in 13 patients (2.8\%) with bilateral calculi during the same session. The patients had solitary kidney $(n=18)$, kyphoscoliosis $(n=7)$, horseshoe kidney $(n=6)$, rotation anomaly $(n=5)$, duplicated collecting system $(n=4)$ and pelvic kidney $(n=2)$. During the surgery, access sheath could not pass, and operation was performed without access sheath in $64(14.1 \%)$ of the patients. There were $26(5.7 \%)$ patients who semirigid ureterorenoscopy could not be advanced bladder and ureter due to stricture, abnormality of the prostatic urethra and/or bladder neck even in young patients. For these cases, ureteral Double J catheter was put in then RIRS was carried out 2-4 weeks later. No balloon dilation was applied to any patients.

Mean operation time was 70.6 (range 45-150) minutes. Mean operation times according to the location of stones were as follows: lower pole 78.1 (55-150) minutes, upper pole 57.3 (45-90) minutes, middle pole 67.4 (60-90) minutes, pelvis 69.9 (40-150) minutes and multiple calyces 71.9 (45-150) minutes. The mean duration of fluoroscopy and hospital stay was 17.6 (3-55) second and 1.3 (1-5) days, respectively. There were a total of twenty (4.4\%) minor complications, which included thirteen patients with infection and/or fever $\left(>38^{\circ} \mathrm{C}\right)$ were hospitalized for about five days and treated with antipyretics and appropriate antibiotics according to their urinary culture results. Other remain-

Table 2. Demographic characteristics of the stones

\begin{tabular}{lc}
\hline Parameters & Values n (\%) \\
\hline Stone Diameter & \\
$\quad 10-20 \mathrm{~mm}$ & $405(89.6)$ \\
$\quad<10 \mathrm{~mm}$ & $47(10.3)$ \\
Stone location & \\
$\quad$ Pelvis & $153(33.8)$ \\
$\quad$ Lower pole & $116(25.6)$ \\
Upper and middle pole & $95(21)$ \\
$\quad$ Multiple calyces & $88(19.4)$ \\
Number of stones & \\
$\quad$ Single & $313(69.2)$ \\
$\quad$ Multiple & $139(30.7)$ \\
Stone side & \\
Left & $206(45.5)$ \\
$\quad$ Right & $233(51.5)$ \\
Bilateral & $13(2.8)$ \\
Stone opacity & \\
Radiopaque or poor radiopacity & $432(95.5)$ \\
Non-opaque & $20(4.4)$ \\
Hounsfield units, average (range) & $898.3(580-1270)$ \\
\hline
\end{tabular}


ing seven patients suffered from renal colic and hematuria treated with oral analgesics. No major complications occurred. No blood transfusion was required. There were no admissions to intensive care or deaths. At postoperative first month follow up, complete stone-free rates and insignificant residual fragments $(<3 \mathrm{~mm})$ were achieved in 355 (78.5\%) patients and 48 patients (10.6\%), respectively, while $49(10.8 \%)$ patients with residual stones $>3 \mathrm{~mm}$ in size. These patients with greater than $3 \mathrm{~mm}$ residual stones were managed with second session RIRS $(n=32), P C N L$ $(n=9)$ and conservative medical management $(n=8)$. The operation was ended prematurely due to very hard stone in seven patients, blurred vision secondary to bleeding in 12 patients, inability to approach to stone using flexible ureterorenoscopy in 12 patients and technical fault in two patients. At first month of control, we achieved a total success rate of $89.1 \%(n=403)$. Distribution of success rates according to the location of renal stones was detected as follows: lower pole $76.3 \%$, middle pole $85.6 \%$, pelvis $93.9 \%$, upper pole $94.3 \%$, and multiple calyces $81.5 \%$.

\section{Discussion}

Urinary system stones are the third most common pathological condition following urinary tract infections and prostate diseases. Aim of urinary stone management is achieving the highest stone-free rate with the lowest morbidity. In recent years, the treatment of renal stones changed considerably, and thanks to the advancement of minimally invasive methods, the highest stone-free rates have been achieved with minimal morbidity. Treatment options have varied from open surgery to less invasive standard, mini-ultra mini or micro PCNL, ESWL and RIRS. Among these procedures, the role of RIRS has broadened in the last decade, and f-URS has become a very important alternative in the diagnosis of upper system pathologies and treatment of renal stones. In recent years, with its low morbidity and its use of natural orifice, this technique is accepted as the first-line treatment for renal stones by both patients and surgeons.

The first RIRS series was carried out in 1990 using f-URS in 208 patients with renal stones, following mechanical ureteral dilation performed for one or two weeks with resultant $87 \%$ stone-free rates ${ }^{[8]}$. Today, ureterorenoscopy management of renal calculi provides an alternative to SWL or PCNL, potentially achieving higher stone-free rates than SWL with lower morbidity than PCNL. Although RIRS is usually recommended as first-line management for renal calculus up to $20 \mathrm{~mm}$, it allows for the treatment of increasingly larger stones in kidney, thus decreasing the role of PCNL and open surgery. Recent studies reported that stone-free rates above $90 \%$ for RIRS in the management of renal stones and as high as $85 \%$ for management of lower pole stones ${ }^{[9]}$. Even in complicated cases, such as stones in chronic kidney disease patients or stones larger than 20 $\mathrm{mm}$, high stone-free rate and safety have been reported [10]. Although RIRS can also be safely used in stones larger than $20 \mathrm{~mm}$, we did not evaluate patients with larger than $20 \mathrm{~mm}$ renal stones.

Double-J stenting, as an end part of the procedure, is still controversial and is frequently recommended to place at the end of the operation. Wu et al. have suggested that a Double-J catheter should be placed in all patients if an access sheath has already been placed ${ }^{[11]}$. When the absence of trauma and no residual stone were present, some authors have reported that stent is not needed to place and that no complication was occurred ${ }^{[12]}$. They recommended that it is not necessary to place a ureteral stent routinely after uncomplicated ureterorenoscopic lithotripsy ${ }^{[12]}$. To reduce mucosal edema and facilitate small stone fragment passage, we have preferred to place a Double-J catheter in our all cases at the end of the operation.

Watterson et al. performed F-URS in 25 patients with bleeding diathesis who were receiving antiaggregant and anticoagulant therapy with $96 \%$ success and noticed the development of retroperitoneal hematoma during the postoperative session which needed blood transfusion ${ }^{[13]}$. In these patients, authors underlined that antiaggregant and/or anticoagulant therapy should be stopped in patients planned for PNL or ESWL, and for these patients, RIRS was a safe and effective treatment option. Unlike this study, RIRS was successfully carried out on 25 patients with renal stones without discontinuance of anticoagulant or antiaggregant therapy. No complication was seen in our presented study. We think that anticoagulant and/or antiaggregant treatment does not have an effect on the treatment of renal stone success rates.

Nowadays, in complicated and challenging patients with bleeding diathesis, urinary diversion, morbid obesity, horseshoe and pelvic kidney, calyceal diverticula, polycystic kidney, lower pole stones, non-opaque and ESWL refractory stones, RIRS has been choosing as a first-line treatment ${ }^{[14]}$. When reviewing the literature, Eryildirim et al. retrospectively evaluated the results of RIRS they carried out on 50 cases with horseshoe kidneys ${ }^{[15]}$. Astolfi et al. performed RIRS on 13 patients with fusion and ectopic renal anomalies ${ }^{[16]}$. In both studies, RIRS was indicated as a safe and feasible choice for the treatment of kidney stones 
in patients with renal ectopic and fusion anomalies. In our study, RIRS was performed in complicated cases with solitary kidney $(n=18)$, kyphoscoliosis $(n=7)$, horseshoe kidneys $(n=6)$, renal malrotations $(n=5)$, duplicated collecting systems $(n=4)$, and pelvic kidneys $(n=2)$. We did not encounter any complications in these patients. Thus, we believe that the presence of renal anomalies does not affect success rates.

Percutaneous nephrolithotomy has its own limitations and possible complications, including fever, sepsis, pneumothorax, colonic injury and hemorrhage requiring blood transfusion ${ }^{[17]}$. On the contrary, in many studies, it was reported that major complications were seen rare, while RIRS complications were seen as lower in Clavien degrees ${ }^{[17]}$. Considering complication rates, RIRS may have a lower risk of severe complications compared to PCNL because the instruments used are of lower caliber and flexible, and operation is performed through direct vision. Bryniarski et al. ${ }^{[18]}$ have assessed outcomes after RIRS and PCNL. They have found that transfusion required in 13 of the PCNL patient group and no transfusion in the RIRS patients group ${ }^{[18]}$. Serious complications are not frequently occurred following RIRS compared to other techniques. The most common complication following RIRS is fever and infection, as is seen in other endourological interventions, while the most serious one is a ureteral stricture. Most of these patients with infection and fever were treated with antipyretics without change or the addition of appropriate antibiotics. Postoperative fever may occur even with sterile preoperative urine and appropriate antibiotic prophylaxis. We think that positive preoperative urine culture, irrigation rate and speed, and operative time are factors that may affect the occurrence of complications. In our study, we believe that since all patients applied appropriate antibiotic prophylaxis, a serious infection was not encountered. Only 13 patients had a high fever $\left(>38^{\circ} \mathrm{C}\right)$ on the postoperative first day, which was relieved with appropriate antibiotherapy according to urine culture results. No patients experienced severe complications, and there were no admissions to intensive care or deaths in the presented series. The results of our study showed a total complication rate (4.4\%) lower than previous reports (16.6-27.4\%) in the literature ${ }^{[19,20]}$.

Routine use of ureteral access sheath during RIRS is still under discussion. Access sheath facilitates repeated passage of the ureteroscope, minimizes damage to ureter, improves the flow of irrigation fluid and visualization within the urethra, and reduces operative times. In addition, using UAS prevents the breaking and damaging of ureterorenoscopy and prolonged its life. This can im- prove both the effectiveness of the surgery and reduce the costs. In this context, in a study where the effectiveness of access sheaths was evaluated, its routine intraoperative use during RIRS was recommended in that it decreases costs and duration of operations, and causes minimal morbidity ${ }^{[14]}$. In another study by Kourambas et al., ${ }^{[21]}$ the effectiveness of access sheaths was assessed, and the authors suggested routine use of access sheaths with its advantages of decreased morbidity and expenditures. However, placement of access sheath can carry an increased risk of ureteral wall ischemia and injury to mucosal or muscular layers of the ureter and a theoretically enhanced risk of ureteral narrowing related to the dimensions. Another disadvantage of access sheath is the development of postoperative ureteral edema. In a study, intrapelvic pressure during f-URS with and without UAS and found higher pressures without using the UAS group [22]. According to this study, they concluded that UAS is potentially protective against pyelovenous and pyelolymphatic backflow with clinical implications for the ureteroscopic management of struvite stones or calculi associated with the urinary tract infection ${ }^{[22]}$. Owing to its above-mentioned advantages, we preferred to use access sheaths for most of our patients and did not encounter any sepsis-related events. We propose strict aseptic precautions, plasma sterilization, negative urine culture and low intrapelvic pressures due to mostly using UAS as the factors responsible for our low infection rates.

Many authors reported that RIRS as reliable and effective treatment technique in the management of renal stones. In the literature, success rates have been reported to range between 65 and 100 percent ${ }^{[14]}$. Takazawa reported treatment of 2-4 cm renal stone handling RIRS and reached a stone-free rate of $100 \%{ }^{[23]}$. Similarly, Prabhakar showed that RIRS could achieve an ultimate $100 \%$ stone-free rate in treating renal calculi with an average diameter of $25 \mathrm{~mm}$ after a single or staged procedure ${ }^{[24]}$. Success rates calculated in our study $(78.4 \%)$ and complied with the success rates that have been reported in the literature.

Our study has some limitations, including its retrospective design in a single-center, the definition of stone-free is a fragment no more than $3 \mathrm{~mm}$, not no stones and lack of any comparison with any other renal stone management modality. In addition, although the same surgical techniques were used in all cases, different surgeons involved in the procedures, and history of previous stone surgery was not evaluated in the analysis of results, which can be presumed as a limitation. 


\section{Conclusion}

We think that as an outcome of our study, in line with advanced technology and increased experience together with its minimal morbidity and high success rates of RIRS and laser lithotripsy will play a gradually evolving role in the treatment of kidney stones smaller than $2 \mathrm{~cm}$. We also believe that our results should be confirmed by further prospective randomized controlled trials conducted in multicenter.

Ethics Committee Approval: Retrospective study.

Peer-review: Externally peer-reviewed.

Authorship Contributions: Concept: R.T.; Design: R.T.; Data Collection or Processing: R.T., A.Ü.; Analysis or Interpretation: R.T., A.Ü.; Literature Search: R.T., A.Ü.; Writing: R.T.

Conflict of Interest: None declared.

Financial Disclosure: The authors declared that this study received no financial support.

\section{References}

1. Romero V, Akpinar H, Assimos DG. Kidney stones: A global picture of prevalence, incidence, and associated risk factors. Rev Urol 2010;12:e86-96.

2. Curhan GC, Rimm EB, Willett WC, Stampfer MJ. Regional variation in nephrolithiasis incidence among United States men. J Urol 1994;151:838-41. [CrossRef]

3. Akinci M, Esen T, Tellaloğlu S. Urinary stone disease in Turkey: an updated epidemiological study. Eur Urol 1991;20:200-3.

4. Argyropoulos AN, Tolley DA. Evaluation of outcome following lithotripsy. Curr Opin Urol 2010;20:154-8. [CrossRef]

5. Van Cleynenbreugel B, Kılıç Ö, Akand M. Retrograde intrarenal surgery for renal stones - Part 1. Turk J Urol 2017;43:112-21.

6. Turk C, Knoll T, Petrik A, Sarica K, Skolarikos A, Straub M, et al. Guidelines on urolithiasis. European association of urology 2017;1-57.

7. Breda A, Angerri O. Retrograde intrarenal surgery for kidney stones larger than $2.5 \mathrm{~cm}$. Curr Opin Urol 2014;24179-83.

8. Fuchs GJ, Fuchs AM. Flexible endoscopy of the upper urinary tract. A new minimally invasive method for diagnosis and treatment. Urologe A 1990;29:313-20.

9. Polat F, Yesil S. Retrograd intrarenal surgery by flexible ureteroscope in patients with spinal deformities. J Spinal Cord Med 2017;40:26-9. [CrossRef]

10. Giusti G, Proietti S, Peschechera R, Taverna G, Sortino G, Cindolo $L$, et al. Sky is no limit for ureteroscopy: extending the indications and special circumstances. World J Urol 2015;33:257-
73. [CrossRef]

11. Wu NZ, Auge BK, Preminger GM. Simplified ureteral stent placement with the assistance of a ureteral access sheath. J Urol 2001;166:206-8. [CrossRef]

12. Chen YT, Chen J, Wong WY, Yang SS, Hsieh CH, Wang CC.Is ureteral stenting necessary after uncomplicated ureteroscopic lithotripsy? A prospective, randomized controlled trial. J Urol 2002;167:1977-80. [CrossRef]

13. Watterson JD, Girvan AR, Cook AJ, Beiko DT, Nott L, Auge BK, et al. Safety and efficacy of holmium: YAG laser lithotripsy in patients with bleeding diatheses. J Urol 2002;168:442-5.

14. Elbir F, Başıbüyük I, Topaktaş R, Kardaş S, Tosun M, Tepeler A, et al. Flexible ureterorenoscopy results: Analysis of 279 cases. Turk J Urol 2015;41:113-8. [CrossRef]

15. Eryildirim B, Kucuk EV, Atis G, Ozturk M, Senkul T, Tuncer M, et al. Safety and efficacy of PNL vs RIRS in the management of stones located in horseshoe kidneys: A critical comparative evaluation. Arch Ital Urol Androl 2018;90:149-54. [CrossRef]

16. Astolfi RH, Freschi G, Berti FF, Gattas N, Molina WR Junior, Meller A. Flexible ureterorenoscopy in position or fusion anomaly: Is it feasible? Rev Assoc Med Bras (1992) 2017;63:685-8. [CrossRef]

17. Parikh KP, Jain RJ, Kandarp AP. Is retrograde intrarenal surgery the game changer in the management of upper tract calculi? A single-center single-surgeon experience of 131 cases. Urol Ann 2018;10:29-34. [CrossRef]

18. Bryniarski P, Stelmach P, Taborowski P, Rajwa P, Adamkiewicz M, Życzkowski $M$, et al. Percutaneous nephrolithotomy with amplatz and alken dilators: an eight-year single tertiary care centre experience. Med Sci Monit 2016;22:4918-23. [CrossRef]

19. Giusti G, Proietti S, Cindolo L, Peschechera R, Sortino G, Berardinelli $F$, et al. Is retrograde intrarenal surgery a viable treatment option for renal stones in patients with solitary kidney? World J Urol 2015;33:309-14. [CrossRef]

20. Gao X, Peng Y, Shi X, Li L, Zhou T, Xu B, et al. Safety and efficacy of retrograde intrarenal surgery for renal stones in patients with a solitary kidney: a single-center experience. J Endourol 2014;28:1290-4. [CrossRef]

21. Kourambas J, Byrne RR, Preminger GM. Does a ureteral access sheath facilitate ureteroscopy? J Urol 2001;165:789-93.

22. Auge BK, Pietrow PK, Lallas CD, Raj GV, Santa-Cruz RW, Preminger GM. Ureteral access sheath provides protection against elevated renal pressures during routine flexible ureteroscopic stone manipulation. J Endourol 2004;18:33-6.

23. Takazawa R, Kitayama S, Tsujii T. Successful outcome of flexible ureteroscopy with holmium laser lithotripsy for renal stones 2 cm or greater. Int J Urol 2012;19:264-7. [CrossRef]

24. Prabhakar M. Retrograde ureteroscopic intrarenal surgery for large $(1.6-3.5 \mathrm{~cm})$ upper ureteric-renal calculus. Indian J Urol 2010;26:46-9. [CrossRef] 